\title{
High Systolic and Diastolic Blood Pressure Variability Is Correlated with the Occurrence of Peripheral Arterial Disease in the First Decade following a Diagnosis of Type 2 Diabetes Mellitus: A New Biomarker from Old Measurement
}

\author{
Chi-Hsiao Yeh, ${ }^{1,2}$ Hsiu-Chin Yu, ${ }^{3}$ Tzu-Yen Huang, ${ }^{1}$ Pin-Fu Huang, ${ }^{1}$ Yao-Chang Wang, \\ Tzu-Ping Chen, ${ }^{1}$ and Shun-Ying Yin ${ }^{1}$ \\ ${ }^{1}$ Department of Thoracic and Cardiovascular Surgery, Chang Gung Memorial Hospital, Keelung, Taiwan \\ ${ }^{2}$ College of Medicine, Chang Gung University, Tao-Yuan, Taiwan \\ ${ }^{3}$ Department of Nursing, Chang Gung Memorial Hospital, Keelung, Taiwan
}

Correspondence should be addressed to Chi-Hsiao Yeh; yehccl@cgmh.org.tw

Received 8 August 2016; Revised 22 September 2016; Accepted 27 September 2016

Academic Editor: Raffaele Serra

Copyright (C) 2016 Chi-Hsiao Yeh et al. This is an open access article distributed under the Creative Commons Attribution License, which permits unrestricted use, distribution, and reproduction in any medium, provided the original work is properly cited.

\begin{abstract}
Background. To assess whether the visit-to-visit variability in blood pressure (BP) is a risk factor of peripheral arterial disease (PAD) in patients with type 2 diabetes mellitus (T2DM) 10 years after diagnosis. Methods. The electronic medical records of 825 patients, who were diagnosed with type 2 diabetes mellitus (T2DM) during 2000-2002 and regularly followed for 10 years, were retrospectively reviewed. A total of 53,284 clinic visit records, including analysis of BP, BMI, serum glycohemoglobin, and lipid profile, were analyzed. Results. Patients were categorized into two groups according to their visit-to-visit variability in systolic and diastolic BP (SBP and DBP, resp.). The high-risk group included patients with high SBP and DBP visit-to-visit variability; this group had a 1.679-fold (95\% CI: 1.141-2.472, $P=0.009)$ increased risk of PAD compared with patients in the low-risk group. Cox regression analysis also demonstrated that the age at which the patients were diagnosed with T2DM, smoking status, and mean creatinine level was significantly associated with increased risk of PAD with a hazard ration of 1.064 (95\% CI: 1.043-1.084, $P<0.001$ ), 1.803 (95\% CI: 1.160-2.804, $P=0.009$ ), and 1.208 (95\% CI: 1.042-1.401, $P=0.012)$, respectively. Conclusions. High SBP and DBP visit-to-visit variability is correlated with PAD in the first decade following a diagnosis of T2DM.
\end{abstract}

\section{Introduction}

In patients with diabetes mellitus, peripheral arterial disease (PAD) is a major risk factor for lower-extremity amputation [1]. However, it is difficult to determine the prevalence of PAD in patients with diabetes given its asymptomatic characteristic, the diverse screening modalities employed, and blunted pain sensation due to peripheral neuropathy, resulting in its underestimation [1]. The ankle-brachial index (ABI), the ratio of the systolic blood pressure (SBP) measured at the ankle to that measured at the brachial artery [2], has a sensitivity of $95 \%$ and specificity of almost $100 \%$ for PAD diagnosis, when validated against angiographically confirmed disease [3]. In diabetic patients older than 40 years of age examined using the $\mathrm{ABI}$, the prevalence of PAD was $20 \%$ [4]. In contrast,
Hirsch et al. [5] reported a prevalence of PAD of $29 \%$ in diabetic patients older than 50 years of age.

Identifying the biomarkers for PAD is important for development of prevention modalities in diabetic patients, especially given that the 5-year cardiovascular event rate, including nonfatal myocardial infarction (MI) and stroke, for PAD patients with T2DM was $20 \%$ and the mortality rate was 30\% [6]. Moreover, after adjusting for risk factors, patients with PAD had a twofold increased risk of MI, stroke, and mortality rate [7]. Moreover, $27 \%$ of patients with PAD demonstrate progression of symptoms with $4 \%$ experiencing limb loss after 5 years [8].

A diagnosis of PAD can identify patients who have higher risk of subsequent $\mathrm{MI}$ or stroke, and treating hypertension in PAD patients reduces the risk of MI, stroke, heart failure, 
and death [9]. Diagnosis and treatment of symptomatic PAD patients with a supervised exercise program and cilostazol may improve the quality of life and prevent functional disability and limb loss as well [10]. Both the American Diabetes Association guidelines [11] and the seventh report of the Joint National Committee on Prevention, Detection, Evaluation, and Treatment of High Blood Pressure [12] recommend using absolute BP as a therapeutic target to prevent clinical stroke and heart disease, as well as PAD with paucity of evidence [13]. However, no randomized prospective clinical trial has conclusively proven the benefits of treatment in individuals with stage 1 systolic hypertension [12].

Although mean BP values are largely considered the cause of the adverse cardiovascular consequences associated with hypertension, the possible role of increased BP variability has also been reported in observational studies and clinical trials [14-17]. Specifically, the visit-to-visit variability of systolic BP (SBP) had been shown to be a novel biomarker for the development of stroke and coronary artery diseases [14-19], the progression of a carotid artery stenosis and peripheral vascular disease [20-22], and the deterioration in renal function for stages 3-4 diabetic chronic kidney disease (CKD) patients [23]. However, little is known about the longterm association of BP visit-to-visit variability with PAD occurrence in diabetic patients with normal ABI at diagnosis.

The association between BP visit-to-visit variability and cardiovascular events generally considers BP measurements at a few time points and in a short to medium follow-up period, limiting the appreciation of the full impact of BP variability on PAD, especially for diabetic patients. Therefore, we evaluated the long-term relationship between BP visit-tovisit variability and the occurrence of PAD in patients from the beginning of their diagnosis with type 2 DM (T2DM).

\section{Materials and Methods}

2.1. Patients and Study Design. We retrospectively collected the following 10-year measurements obtained at every outpatient clinic visit of 825 patients who were first diagnosed with T2DM during 2000-2002 at Chang Gung Memorial Hospital, Keelung: blood pressure, body weight, body height, and laboratory data. T2DM was diagnosed in accordance with the criteria of the American Diabetes Association [24]. Body mass index (BMI) was defined as weight (kilograms) divided by height (meters) squared. Patients were classified as nonsmokers, former smokers, or current smokers according to the electronic medical record. PAD was diagnosed on the basis of an $\mathrm{ABI} \leq 0.9$ [25]. Patients who developed PAD or cardiovascular disease (CVD), including coronary artery disease, MI, ischemic stroke, or transient ischemic attack [26], before being diagnosed with diabetes were excluded. Patients who never had an ABI assessment during the 10-year follow-up period, those without PAD who did not have complete ABI data in the 10th year of follow-up, and patients with less than 10-year follow-up were also excluded. Dyslipidemia was defined as without treatment, total cholesterol levels $>200 \mathrm{mg} / \mathrm{dL}$, low-density lipoprotein cholesterol levels $>100 \mathrm{mg} / \mathrm{dL}$, high-density lipoprotein cholesterol levels $<50 \mathrm{mg} / \mathrm{dL}$ in females and $<40 \mathrm{mg} / \mathrm{dL}$ in males, or triglycerides $>150 \mathrm{mg} / \mathrm{dL}$. Hypertension was defined as SBP $\geq 130 \mathrm{mmHg}$ or diastolic blood pressure (DBP) $\geq 80 \mathrm{mmHg}$ in diabetic patient before any treatment was initiated [12].

This study was conducted in accordance with the Declaration of Helsinki and was approved by the Institutional Review Board of Chang Gung Memorial Hospital; informed consent was waived.

2.2. Laboratory Assessments. BP measurements at every visit were recorded throughout the follow-up period till PAD was diagnosed. All outpatient clinics used automated sphygmomanometers operated by trained medical assistants after 1015 minutes resting, with repeated measurements performed as needed by physicians using aneroid sphygmomanometers [27]. Fasting serum total cholesterol, low-density lipoprotein, high-density lipoprotein, and triglyceride concentrations were assessed using standard enzymatic methods. Hemoglobin $A_{1 c}$ was assayed using high-performance liquid chromatography and expressed with the unit defined by the National Glycohemoglobin Standardization Program.

2.3. Definition of BP Visit-To-Visit Variability. BP visit-to-visit variability and the coefficient of variation (standard deviation (SD) of mean BP divided by mean BP) of SBP and DBP were determined [28]. The BP instability index was expressed as the delta BP, which was defined as a difference between the maximum $\mathrm{BP}$ and the minimum $\mathrm{BP}$, through all visits till $\mathrm{PAD}$ was diagnosed [29].

2.4. Statistical Analysis. Means and frequencies of potential confounding variables were calculated. The relationships between variability in SBP and DBP, as well as other variables, and PAD were examined by Pearson's correlation analyses. To examine the effects of various factors on the occurrence of $\mathrm{PAD}$, the following factors were considered simultaneously as independent variables for Cox multiple regression analysis: age at DM diagnosis, sex, BMI, average SBP and DBP, SD of SBP and DBP, maximum of SBP and DBP, delta SBP, delta DBP, hemoglobin $A_{1 c}$, total cholesterol, triglyceride, smoking status, presence of CVD, hypertension, and dyslipidemia. All continuous variables are presented as the mean \pm SD or absolute number. A $P$ value $<0.05$ was considered statistically significant. The area under each receiver operating curve and 95\% confidence intervals (CI) were estimated to compare the relative ability of the SD of SBP and DBP to identify risk of peripheral arterial disease in diabetic patients. The optimal cut-point BP was calculated based on the Youden Index [30], which was calculated as sensitivity + specificity 1 [31]. Multicollinearity was assessed using variance inflation factor (VIF) among average, SD, maximum, and grouping of VVV of SBP and DBP [32, 33]. The power calculation was performed with pass software. Multicollinearity was diagnosed with a VIF, one of the most common tools used by statisticians, of 5 and above $[32,33]$.

\section{Results}

Nine hundred and thirty-six patients were first diagnosed with DM at Chang Gung Memorial Hospital from 2000 to 
2002. Sixty-nine patients who died or were lost to followup were excluded. Twenty-three patients without final ABI data and 19 patients who had PAD or CVD before their DM diagnosis were also excluded. The characteristics of the 825 patients enrolled in this study are shown in Table 1. The overall mean age at diagnosis with DM was $53.6 \pm 10.5$ years. At baseline, the ABI for all patients was in the range of $0.9-$ 1.3. The median observation period was $148.1 \pm 16.0$ months. At the end of the observation period, the right and left leg $\mathrm{ABI}$ levels for all patients were $1.02 \pm 0.24$ and $1.02 \pm 0.42$, respectively. There were 114 patients diagnosed with PAD during the 10-year follow-up with an average time of PAD diagnosis of $116.7 \pm 12.8$ months after their DM diagnosis. The average SBP and DBP for all patients was $136.8 \pm 10.2$ and $73.5 \pm 6.4 \mathrm{mmHg}$, respectively. The SD of the SBP and DBP for all patients was $14.8 \pm 3.8$ and $7.5 \pm 2.1 \mathrm{mmHg}$, respectively.

Multivariate Cox regression analyses revealed that the SD of SBP was positively correlated with the occurrence of PAD $(P=0.037$; Table 2$)$. However, the maximum of SBP and delta of SBP were not significantly correlated. The mean, SD, maximum, and delta of DBP were not significantly correlated with the occurrence of PAD. In addition to the SD of SBP, the age at DM diagnosis was positively correlated with the occurrence of renal function impairment $(P<0.001, \mathrm{HR}=$ $1.064,95 \% \mathrm{CI}=1.043-1.084)$. In addition, the occurrence of PAD was associated with mean creatinine level $(P=0.012)$ and current smoking status $(P=0.009)$. However, mean or $\mathrm{SD}$ of hemoglobin $\mathrm{A}_{1 \mathrm{c}}$, BMI, total cholesterol, low-density lipoprotein, high-density lipoprotein, and triglyceride were not independently correlated with the occurrence of PAD (Table 2).

We next categorized the patients into high- or low-risk groups on the basis of their SD of SBP or DBP. Cut-off points for the SD of SBP and DBP, where the sensitivity approximates specificity for the occurrence of $\mathrm{PAD}$, are 16.3 and $7.6 \mathrm{mmHg}$, respectively. Patients with SD of SBP and DBP higher than the cut-off values $(n=199)$ were placed in the high-risk group, and all the other patients $(n=626)$ were in the lowrisk group. The multicollinearity was assessed between VVV grouping and mean, SD, maximum, and delta of SBP and DBP. The VIFs of all these factors were less than 2 (Table 2), which represented the idea that the grouping according to the VVV was independently different factor among these parameters. The characteristics of both groups were shown in Table 3. The age at DM diagnosis, hypertension history, SD of BMI, average SBP and DBP, SD of SBP and DBP, delta SBP and DBP, mean and SD of hemoglobin $\mathrm{A}_{1 \mathrm{c}}$, mean total cholesterol, and mean and SD of creatinine level were significantly different between the low- and high-risk groups $(P \leq 0.034)$.

In the 10 years following their DM diagnosis, 50 patients (25.1\%) in the high-risk group had PAD versus 64 patients $(10.2 \%)$ in the low-risk group $(P<0.001$; Table 3$)$. The PADfree survival curve between patients in high- and low-risk groups was shown in Figure 1. In addition, the occurrence of CVD in the high-risk group was significantly higher than that of the low-risk group (25.1\% versus $11.7 \%$; $P<0.001)$.

Cox multivariate regression analysis revealed that the risk of PAD increased by 1.064 -fold as the age at diagnosis
TABLE 1: Characteristics of the study participants $(n=825)$.

\begin{tabular}{|c|c|}
\hline Characteristic & \\
\hline Age at DM diagnosis (y) & $53.6 \pm 10.5$ \\
\hline Sex (male, \%) & $390(47.3)$ \\
\hline Smoking status (none/former/current) & $628 / 49 / 148$ \\
\hline Hypertension (\%) & $629(76.2)$ \\
\hline Hyperlipidemia (\%) & $793(96.1)$ \\
\hline Body mass index $\left(\mathrm{kg} / \mathrm{m}^{2}\right)$ & $26.8 \pm 3.9$ \\
\hline Number of measurements & $45.0 \pm 24.8$ \\
\hline Average SBP (mmHg) & $136.8 \pm 10.2$ \\
\hline Number of measurements & $63.4 \pm 29.4$ \\
\hline SD of SBP $(\mathrm{mmHg})$ & $14.8 \pm 3.8$ \\
\hline Maximum of SBP (mmHg) & $174.5 \pm 17.5$ \\
\hline Delta SBP (mmHg) & $72.5 \pm 25.6$ \\
\hline Average DBP (mmHg) & $73.5 \pm 6.4$ \\
\hline SD of DBP (mmHg) & $7.5 \pm 2.1$ \\
\hline Maximum of DBP (mmHg) & $93.8 \pm 11.3$ \\
\hline Delta DBP (mmHg) & $38.2 \pm 14.6$ \\
\hline Hemoglobin $\mathrm{A}_{1 \mathrm{c}}(\%)$ & $7.6 \pm 1.0$ \\
\hline Number of measurements & $34.6 \pm 10.9$ \\
\hline Total cholesterol (mg/dL) & $193.5 \pm 28.6$ \\
\hline Number of measurements & $11.9 \pm 6.1$ \\
\hline High-density lipoprotein (mg/dL) & $38.5 \pm 10.6$ \\
\hline Low-density lipoprotein (mg/dL) & $118.5 \pm 20.0$ \\
\hline Triglyceride $(\mathrm{mg} / \mathrm{dL})$ & $150.1 \pm 112.1$ \\
\hline Initial creatinine $(\mathrm{mg} / \mathrm{dL})$ & $0.76 \pm 0.54$ \\
\hline Average creatinine (mg/dL) & $0.99 \pm 0.71$ \\
\hline Number of measurements & $17.9 \pm 6.7$ \\
\hline \multicolumn{2}{|c|}{ Clinical events during the 10 -year follow-up } \\
\hline $\operatorname{PAD}(\%)$ & $114(13.8)$ \\
\hline $\mathrm{CVD}^{\mathrm{a}}(\%)$ & $123(14.9)$ \\
\hline CAD or MI (\%) & $38(4.6)$ \\
\hline TIA or stroke (\%) & $88(10.7)$ \\
\hline Total follow-up period (months) & $148.1 \pm 16.0$ \\
\hline
\end{tabular}

DM, diabetes mellitus; SBP, systolic blood pressure; DBP, diastolic blood pressure; SD, standard deviation; PAD, peripheral arterial disease; CVD, cerebrovascular disease; CAD, coronary artery disease; MI, myocardial infarction; TIA, transient ischemic attack; CKD, chronic kidney disease.

${ }^{a}$ Defined as cerebrovascular disease, neurodegenerative disease, and Parkinson's disease that required medical treatment and long-term follow-up.

increased by 1 year $(P<0.001,95 \%$ CI 1.043-1.084; Table 4). High BP visit-to-visit variability also increased likelihood of PAD within 10 years of being diagnosed with DM by 1.679fold $(P=0.009)$. Current smoking status and elevation of mean creatinine level was also associated with increased risk of PAD (Table 4).

\section{Discussion}

This study showed that patients with high visit-to-visit variability in both SBP and DBP were more frequently diagnosed with PAD in the first decade following diagnosis with DM. However, the mean and delta SBP/DBP, mean serum lipid profile, mean and $\mathrm{SD}$ of hemoglobin $\mathrm{A}_{1 \mathrm{c}}$ concentration, and 
TABLE 2: Multivariate Cox regression analyses of the factors associated with peripheral arterial disease $(n=825)$ in the 10 years following a diagnosis of DM.

\begin{tabular}{|c|c|c|c|c|}
\hline Independent variable & Hazard ratio & $95 \% \mathrm{CI}$ & VIF & $P$ value \\
\hline Sex $($ female $=0)$ & 0.893 & $0.558-1.429$ & & 0.636 \\
\hline Age at DM diagnosis & 1.069 & $1.043-1.095$ & & $<0.001$ \\
\hline Nonsmoking & & & & 0.026 \\
\hline Former smoker & 1.627 & $0.765-3.460$ & & 0.206 \\
\hline Current smoker & 2.070 & $1.212-3.536$ & & 0.008 \\
\hline Hypertension & 1.426 & $0.733-2.774$ & & 0.296 \\
\hline Dyslipidemia & 4.124 & $0.543-31.319$ & & 0.171 \\
\hline Mean SBP & 1.008 & $0.974-1.044$ & 1.420 & 0.639 \\
\hline SD of SBP & 1.088 & $0.970-1.220$ & 1.816 & 0.150 \\
\hline Maximum of SBP & 0.984 & $0.960-1.008$ & 1.267 & 0.182 \\
\hline Delta of SBP & 1.001 & $0.986-1.015$ & 1.806 & 0.932 \\
\hline Mean DBP & 0.996 & $0.936-1.060$ & 1.460 & 0.899 \\
\hline SD of DBP & 0.940 & $0.757-1.168$ & 1.859 & 0.578 \\
\hline Maximum of DBP & 1.015 & $0.982-1.049$ & 1.267 & 0.364 \\
\hline Delta of DBP & 0.997 & $0.971-1.023$ & 1.779 & 0.809 \\
\hline Mean BMI & 0.972 & $0.917-1.030$ & & 0.338 \\
\hline SD of BMI & 1.251 & $0.868-1.802$ & & 0.230 \\
\hline Mean hemoglobin $A_{1 c}$ & 0.979 & $0.764-1.254$ & & 0.864 \\
\hline $\mathrm{SD}$ of hemoglobin $\mathrm{A}_{1 \mathrm{c}}$ & 0.979 & $0.565-1.695$ & & 0.940 \\
\hline Mean serum cholesterol & 1.011 & $0.998-1.023$ & & 0.088 \\
\hline SD of serum cholesterol & 0.994 & $0.983-1.005$ & & 0.258 \\
\hline Mean serum LDL & 1.004 & $0.990-1.018$ & & 0.565 \\
\hline SD of serum LDL & 0.988 & $0.976-1.001$ & & 0.079 \\
\hline Mean serum HDL & 0.993 & $0.968-1.018$ & & 0.575 \\
\hline SD of serum HDL & 1.022 & $0.980-1.067$ & & 0.309 \\
\hline Mean serum triglyceride & 1.001 & $0.996-1.005$ & & 0.815 \\
\hline SD of serum triglyceride & 1000 & $0.995-1.004$ & & 0.892 \\
\hline Mean creatinine & 1.305 & $1.024-1.665$ & & 0.032 \\
\hline SD of creatinine & 0.833 & $0.474-1.461$ & & 0.523 \\
\hline
\end{tabular}

DM, diabetes mellitus; SBP, systolic blood pressure; DBP, diastolic blood pressure; SD, standard deviation; CV, coefficient of variation; BMI, body mass index; LDL, low density lipoprotein; HDL, high density lipoprotein; VIF, variance inflation factor.

BMI were not correlated with the occurrence of PAD. Our results also confirmed that old age at DM diagnosis, smoking, and renal function impairment would increase the risk of PAD in the first decade after DM was diagnosed. Cessation of smoking in patients with PAD could substantially reduce the risk of death, myocardial infarction, and amputation and increase the patency rate of lower-extremity angioplasty and surgical revascularization [25]. Two recent studies revealed that central obesity with elevated BMI was positively associated with $\mathrm{ABI}$ increase $[34,35]$. However, the relationship between BMI and PAD could not be identified from our study.

Assessment of the effects of short-term variability of BP has traditionally dominated this field of research [36] and diminished the interest in long-term variability of BP, such as those occurring between days or months. However, recent studies have shown that long-term visit-to-visit variability of BP may have greater prognostic value than mean BP or shortterm variability $[19,37,38]$. These studies recommended that optimal antihypertension treatment included avoidance of inconsistent BP control and large BP visit-to-visit variability [36].

The effect of high BP variability on PAD occurrence has rarely been studied. Most prospective BP variability studies focused on the cardiovascular mortality and morbidities, including MI, stroke, and heart failure [17, 39-41]. These studies followed patients for a relatively short period of 1.5 to $7.8 \mathrm{y}$, and less than $10 \%$ of the study patients had DM [39-41]. DM is one of the strongest risk factors for critical limb ischemia and amputation, as well as incident PAD in population studies [42]. However, few studies have completely focused on the detrimental cardiovascular effect of BP variability in diabetic patients. Mancia et al. [43] revealed that $\mathrm{DM}$ did predict $\mathrm{ABI}$ decline over an average of $4.6 \mathrm{y}$ of follow-up. Our study proved that high variability of $\mathrm{BP}$ is a risk factor for the occurrence of PAD in the 10 years following DM diagnosis. These diabetic patients with $\mathrm{PAD}$ are at high risk for adverse cardiovascular events unless 
TABLE 3: Demographics and clinical characteristics of the low- and high-risk groups as determined by BP visit-to-visit variability.

\begin{tabular}{|c|c|c|c|}
\hline & Low-risk group $(n=626)$ & High-risk group $(n=199)$ & $P$ value \\
\hline Age at DM diagnosis (y) & $52.5 \pm 10.3$ & $57.2 \pm 10.6$ & $<0.001$ \\
\hline Sex (male, \%) & $296(47.3)$ & $94(47.2)$ & 0.528 \\
\hline Smoking (none/former/current) & $480 / 36 / 110$ & $148 / 13 / 38$ & 0.808 \\
\hline Hypertension (\%) & $449(71.7)$ & $180(90.5)$ & $<0.001$ \\
\hline Hyperlipidemia (\%) & $600(95.8)$ & $193(97.0)$ & 0.313 \\
\hline Body mass index $\left(\mathrm{kg} / \mathrm{m}^{2}\right)$ & $26.8 \pm 3.9$ & $27.0 \pm 3.9$ & 0.521 \\
\hline Number of measurements & $44.8 \pm 24.4$ & $45.4 \pm 26.0$ & 0.772 \\
\hline SD of BMI $\left(\mathrm{kg} / \mathrm{m}^{2}\right)$ & $1.0 \pm 0.5$ & $1.2 \pm 0.6$ & $<0.001$ \\
\hline Average SBP (mmHg) & $135.3 \pm 9.6$ & $141.3 \pm 10.7$ & $<0.001$ \\
\hline Number of measurements & $61.8 \pm 27.6$ & $68.6 \pm 34.1$ & 0.004 \\
\hline SD of SBP $(\mathrm{mmHg})$ & $13.3 \pm 2.6$ & $19.5 \pm 3.3$ & $<0.001$ \\
\hline Maximum of SBP (mmHg) & $170.1 \pm 15.9$ & $188.4 \pm 15.1$ & $<0.001$ \\
\hline Delta SBP (mmHg) & $65.1 \pm 20.0$ & $96.0 \pm 27.1$ & $<0.001$ \\
\hline Average DBP (mmHg) & $73.1 \pm 6.0$ & $74.7 \pm 7.1$ & 0.002 \\
\hline SD of DBP (mmHg) & $6.8 \pm 1.5$ & $9.7 \pm 2.3$ & $<0.001$ \\
\hline Maximum of DBP (mmHg) & $91.3 \pm 9.4$ & $101.9 \pm 12.9$ & $<0.001$ \\
\hline Delta DBP (mmHg) & $34.4 \pm 11.6$ & $50.0 \pm 16.7$ & $<0.001$ \\
\hline Hemoglobin $A_{1 c}(\%)$ & $7.5 \pm 1.0$ & $7.7 \pm 1.1$ & 0.019 \\
\hline Number of measurements & $35.4 \pm 10.7$ & $32.0 \pm 11.3$ & $<0.001$ \\
\hline SD of Hemoglobin $A_{1 c}(\%)$ & $0.9 \pm 0.6$ & $1.1 \pm 0.5$ & $<0.001$ \\
\hline Total cholesterol (mg/dL) & $192.3 \pm 26.7$ & $197.2 \pm 33.7$ & 0.034 \\
\hline Number of measurements & $12.0 \pm 5.8$ & $11.3 \pm 6.9$ & 0.147 \\
\hline High-density lipoprotein (mg/dL) & $38.5 \pm 10.7$ & $38.4 \pm 10.5$ & 0.877 \\
\hline Low-density lipoprotein (mg/dL) & $118.6 \pm 19.7$ & $118.4 \pm 21.0$ & 0.877 \\
\hline Triglyceride (mg/dL) & $147.0 \pm 106.8$ & $159.8 \pm 127.0$ & 0.1640 \\
\hline Average creatinine & $0.89 \pm 0.4$ & $1.3 \pm 1.2$ & $<0.001$ \\
\hline Number of measurements & $13.6 \pm 5.1$ & $15.8 \pm 9.5$ & $<0.001$ \\
\hline SD of creatinine & $0.1 \pm 0.3$ & $0.3 \pm 0.4$ & $<0.001$ \\
\hline \multicolumn{4}{|c|}{ Clinical events during the 10 -year follow-up period } \\
\hline $\mathrm{PAD}(\%)$ & $64(10.2)$ & $50(25.1)$ & $<0.001$ \\
\hline Interval from DM diagnosis (y) & $8.1 \pm 2.0$ & $7.7 \pm 2.5$ & 0.403 \\
\hline $\operatorname{CVD}^{\mathrm{a}}(\%)$ & $73(11.7)$ & $50(25.1)$ & $<0.001$ \\
\hline Interval from DM diagnosis $(\mathrm{y})$ & $5.4 \pm 2.8$ & $4.9 \pm 3.5$ & 0.388 \\
\hline $\mathrm{CAD}$ or $\mathrm{MI}(\%)$ & $24(3.8)$ & $14(7.0)$ & 0.051 \\
\hline Interval from DM diagnosis $(\mathrm{y})$ & $5.4 \pm 2.7$ & $3.7 \pm 3.3$ & 0.094 \\
\hline TIA or stroke $(\%)$ & $51(8.1)$ & $37(18.6)$ & $<0.001$ \\
\hline Interval from DM diagnosis $(\mathrm{y})$ & $5.3 \pm 2.9$ & $5.4 \pm 3.4$ & 0.903 \\
\hline Recurrent TIA or stroke events & $0.2 \pm 0.5$ & $0.4 \pm 0.8$ & 0.152 \\
\hline Total follow-up (months) & $148.6 \pm 15.2$ & $146.4 \pm 18.4$ & 0.098 \\
\hline
\end{tabular}

DM, diabetes mellitus; SBP, systolic blood pressure; DBP, diastolic blood pressure; SD, standard deviation; PAD, peripheral arterial disease; CVD, cerebrovascular disease; CAD, coronary artery disease; MI, myocardial infarction; TIA, transient ischemic attack; CKD, chronic kidney disease.

${ }^{a}$ Defined as cerebrovascular disease, neurodegenerative disease, and parkinsonism that required medical treatment and long-term follow-up.

the PAD is recognized. From the results of the present study, diabetic patients with high BP visit-to-visit variability had a 1.679-fold increased risk of the occurrence of PAD. For the first time, our results demonstrated that a visit-to-visit variability VVV of $\mathrm{SBP}>16.3 \mathrm{mmHg}$ with a visit-to-visit variability of DBP $>7.6 \mathrm{mmHg}$ significantly increased the risk of PAD in the first decade following a diagnosis with $\mathrm{DM}$. The adverse consequences of high $\mathrm{BP}$ variability on the cardiovascular system might result from the traumatic effect of large blood pressure oscillations enhancing the intravascular pressures on the vessel wall, promoting tissue growth and atherosclerosis [44].

Office BP as the measurement of variability of BP may be limited due to white coat hypertension although this issue is controversial. A meta-analysis that included 7961 untreated participants reported that the cardiovascular risk 


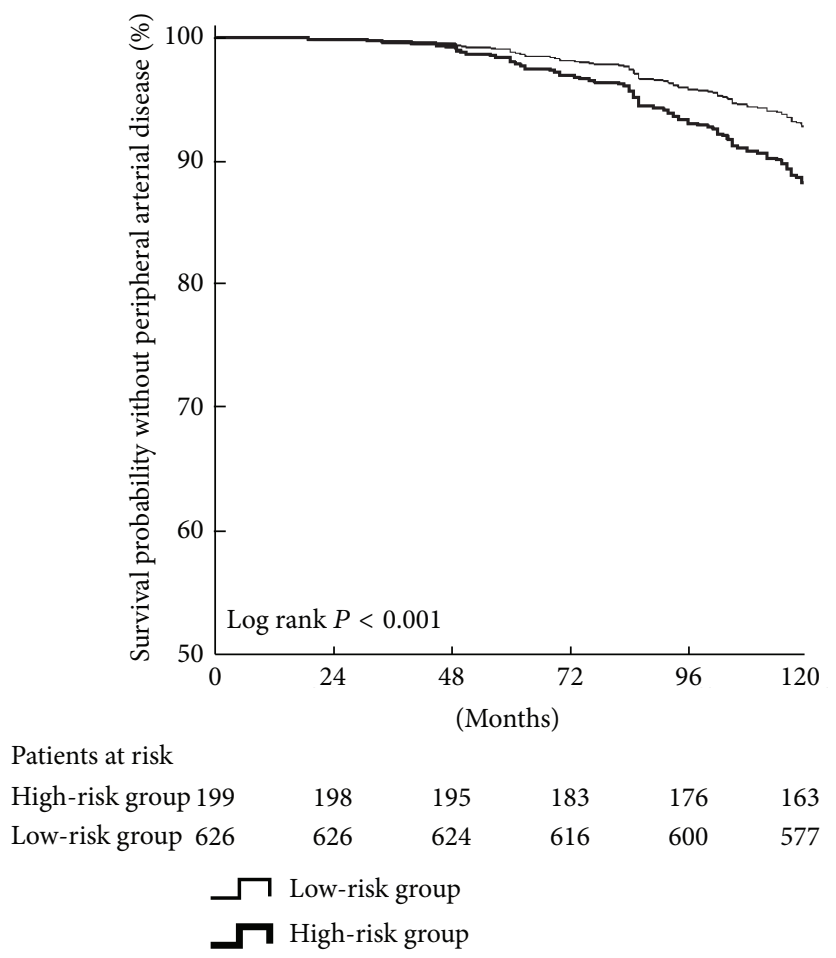

Figure 1: Kaplan-Meier plot of peripheral arterial disease occurrence over 10 years following a diagnosis of type 2 diabetes. Patients were grouped into high- (high BP visit-to-visit variability) and low-risk groups.

TABLE 4: Multivariate Cox regression analysis of factors associated with the occurrence of peripheral arterial disease.

\begin{tabular}{lccc}
\hline & Hazard ratio & $95 \%$ CI & $P$ value \\
\hline Age $(+1$ y) & 1.064 & $1.043-1.084$ & $<0.001$ \\
Nonsmoking & 1 & & \\
Former smoking & 1.645 & $0.771-2.783$ & 0.244 \\
Current smoking & 1.803 & $1.160-2.804$ & 0.009 \\
High SBP and DBP & 1.679 & $1.141-2.472$ & 0.009 \\
visit-to-visit variability & 1.208 & $1.042-1.401$ & 0.012 \\
Mean Creatinine (mg/dL) &
\end{tabular}

$\mathrm{DM}$, diabetes mellitus; SBP, systolic blood pressure; DBP, diastolic blood pressure; CI, confidence interval.

was not significantly different between white coat hypertension and normotension [45]. Another study from the International Database of HOme blood pressure in relation to Cardiovascular Outcome, which followed 6458 patients for a median of $8.3 \mathrm{y}$, reported that the cardiovascular risk for the white coat hypertension did not differ from that of the treated participants [46]. However, another large metaanalysis of 29,100 patients comparing white coat hypertension participants with sustained hypertension participants found that CVD mortality (4.2\%) was significantly lower in white coat hypertension participants $(6.6 \%$; OR $=0.47,95 \%$ CI $0.35-0.64 ; P<0.001)$ than those with sustained hypertension participants [46]. Thus, when office BP is used as the measurement of BP variability, white coat hypertension is not a factor that induces bias.
The retrospective nature of the present study and its sample size are two of the limitations of the present study. The possibility of type 2 error exists. Another limitation is the medication record through the 10-year follow-up period. Antihypertensive drug use in each patient was not consistent throughout the 10-year follow-up period. Thus, it is very difficult to clarify the effect of BP visit-to-visit variability amelioration by each category of antihypertensive drug, such as calcium channel blockers, which had a controversial effect on blunting the amplitude of BP visit-to-visit variability [23]. The other limitation of the present study is the absence of antihypertensive prescription fill data and information regarding patients' adherence to treatment regimens. However, low antihypertensive medication adherence explained only a small proportion of BP visit-to-visit variability [38], which implied that the absence of medication adherence data does not have a major impact on the results of the present study.

Despite the aforementioned limitations, this study has several strengths, including the long follow-up period to assess the occurrence of PAD and the available information on demographic, clinical, and long-term BP data. In addition, the use of an electronic medical record database provided real-world evidence on the status of hypertension control in the first decade following a diabetes diagnosis and minimizes selection bias related to self-selection into the study. Prospective studies are in need in verifying the high variability of SBP visit-to-visit as early biomarker for detection of PAD in the first decade following the diagnosis of T2DM. More research is needed to fully understand the association between $\mathrm{BP}$ 
visit-to-visit variability and risk of vascular events in diabetic patients, and large-scale pooled analyses of multiple cohorts will be required [19].

\section{Conclusion}

The present study showed that, in diabetic patients with initially normal ABI values, high $\mathrm{BP}$ visit-to-visit variability was a significant early biomarker for detection of PAD in the first decade following the diagnosis of T2DM.

\section{Competing Interests}

There are no competing interests to declare.

\section{Acknowledgments}

This study was supported by a Chang Gung Medical Research Program grant of Chang Gung Memorial Hospital (CMRPG 2C0101 and CGRPG2F0041).

\section{References}

[1] American Diabetes Association, "Peripheral arterial disease in people with diabetes," Diabetes Care, vol. 26, no. 12, pp. 33333341, 2003.

[2] V. Aboyans, M. H. Criqui, P. Abraham et al., "Measurement and interpretation of the Ankle-Brachial Index: a scientific statement from the American Heart Association," Circulation, vol. 126, no. 24, pp. 2890-2909, 2012.

[3] E. F. Bernstein and A. Fronek, "Current status of noninvasive tests in the diagnosis of peripheral arterial disease," Surgical Clinics of North America, vol. 62, no. 3, pp. 473-487, 1982.

[4] T. A. Elhadd, R. Robb, R. T. Jung, P. A. Stonebridge, and J. J. F. Belch, "Pilot study of prevalence of asymptomatic peripheral arterial occlusive disease in patients with diabetes attending a hospital clinic," Practical Diabetes International, vol. 16, no. 6, pp. 163-166, 1999.

[5] A. T. Hirsch, M. H. Criqui, D. Treat-Jacobson et al., "Peripheral arterial disease detection, awareness, and treatment in primary care," Journal of the American Medical Association, vol. 286, no. 11, pp. 1317-1324, 2001.

[6] J. I. Weitz, J. Byrne, G. P. Clagett et al., "Diagnosis and treatment of chronic arterial insufficiency of the lower extremities: a critical review," Circulation, vol. 94, no. 11, pp. 3026-3049, 1996.

[7] J. R. Das and R. T. Eberhardt, "Contemporary risk assessment and cardiovascular outcomes in peripheral arterial disease," Cardiovascular and Hematological Disorders-Drug Targets, vol. 13, no. 3, pp. 185-196, 2013.

[8] L. Norgren, W. R. Hiatt, J. A. Dormandy et al., "Inter-society consensus for the management of peripheral arterial disease (TASC II)," Journal of Vascular Surgery, vol. 45, no. 1, supplement, pp. S5-S67, 2007.

[9] J. W. Olin, "Treatment of hypertensive patients with peripheral arterial disease," in The Essentials of High Blood Pressure: Basic Science, Population Science, and Clinical Management, J. L. Izzo Jr. and H. R. Black, Eds., pp. 467-469, Lippincott Williams \& Wilkins, Philadelphia, Pa, USA, 2003.

[10] J. W. Olin and B. A. Sealove, "Peripheral artery disease: current insight into the disease and its diagnosis and management," Mayo Clinic Proceedings, vol. 85, no. 7, pp. 678-692, 2010.
[11] American Diabetes Association, "Cardiovascular disease and risk management. Sec. 8. In Standards of Medical Care in Diabetes 2016," Diabetes Care, vol. 39, supplement 1, pp. S60S71, 2016.

[12] A. V. Chobanian, G. L. Bakris, H. R. Black et al., "National Heart, Lung, and Blood Institute; National High Blood Pressure Education Program Coordinating Committee. Seventh report of the joint national committee on prevention, detection, evaluation, and treatment of high blood pressure," Hypertension, vol. 42, no. 6, pp. 1206-1252, 2003.

[13] R. M. Cooper-DeHoff, Y. Gong, E. M. Handberg et al., "Tight blood pressure control and cardiovascular outcomes among hypertensive patients with diabetes and coronary artery disease," Journal of the American Medical Association, vol. 304, no. 1, pp. 61-68, 2010.

[14] G. Parati, J. E. Ochoa, P. Salvi, C. Lombardi, and G. Bilo, "Prognostic value of blood pressure variability and average blood pressure levels in patients with hypertension and diabetes," Diabetes Care, vol. 36, no. 2, pp. S312-S324, 2013.

[15] Y. Matsui, J. Ishikawa, K. Eguchi, S. Shibasaki, K. Shimada, and K. Kario, "Maximum value of home blood pressure: a novel indicator of target organ damage in hypertension," Hypertension, vol. 57, no. 6, pp. 1087-1093, 2011.

[16] M. Kikuya, T. Ohkubo, H. Metoki et al., "Day-by-day variability of blood pressure and heart rate at home as a novel predictor of prognosis: The Ohasama Study," Hypertension, vol. 52, no. 6, pp. 1045-1050, 2008.

[17] J. K. Johansson, T. J. Niiranen, P. J. Puukka, and A. M. Jula, "Prognostic value of the variability in home-measured blood pressure and heart rate: the Finn-HOME study," Hypertension, vol. 59, no. 2, pp. 212-218, 2012.

[18] E. Budiman-Mak, N. Epstein, M. Brennan et al., "Systolic blood pressure variability and lower extremity amputation in a nonelderly population with diabetes," Diabetes Research and Clinical Practice, vol. 114, pp. 75-82, 2016.

[19] P. M. Rothwell, S. C. Howard, E. Dolan et al., "Prognostic significance of visit-to-visit variability, maximum systolic blood pressure, and episodic hypertension," The Lancet, vol. 375, no. 9718, pp. 895-905, 2010.

[20] J. Hata, H. Arima, P. M. Rothwell et al., "Effects of visit-tovisit variability in systolic blood pressure on macrovascular and microvascular complications in patients with type 2 diabetes mellitus: the advance trial," Circulation, vol.128, no. 12, pp.13251334, 2013.

[21] P. M. Rothwell, S. C. Howard, E. Dolan et al., "Effects of $\beta$ blockers and calcium-channel blockers on within-individual variability in blood pressure and risk of stroke," The Lancet Neurology, vol. 9, no. 5, pp. 469-480, 2010.

[22] K. Eguchi, S. Hoshide, J. E. Schwartz, K. Shimada, and K. Kario, "Visit-to-visit and ambulatory blood pressure variability as predictors of incident cardiovascular events in patients with hypertension," American Journal of Hypertension, vol. 25, no. 9, pp. 962-968, 2012.

[23] K. Yokota, M. Fukuda, Y. Matsui, K. Kario, and K. Kimura, "Visit-to-Visit variability of blood pressure and renal function decline in patients with diabetic chronic kidney disease," Journal of Clinical Hypertension, vol. 16, no. 5, pp. 362-366, 2014.

[24] American Diabetes Association, "Diagnosis and classification of diabetes mellitus," Diabetes Care, vol. 37, supplement 1, pp. S81-S90, 2014.

[25] T. W. Rooke, A. T. Hirsch, S. Misra et al., "2011 ACCF/AHA focused update of the guideline for the management of 
patients with peripheral artery disease (Updating the 2005 Guideline): a report of the american college of cardiology foundation/American Heart Association task force on practice guidelines," Circulation, vol. 124, no. 18, pp. 2020-2045, 2011.

[26] S. Mendis, P. Puska, and B. Norrving, Global Atlas on Cardiovascular Disease Prevention and Control-Policies, Strategies and Interventions, World Health Organization, Geneva, Switzerland, 2011.

[27] M. G. Jaffe, G. A. Lee, J. D. Young, S. Sidney, and A. S. Go, "Improved blood pressure control associated with a large-scale hypertension program," The Journal of the American Medical Association, vol. 310, no. 7, pp. 699-705, 2013.

[28] S. S. Franklin, M. G. Larson, S. A. Khan et al., "Does the relation of blood pressure to coronary heart disease risk change with aging?: the Framingham Heart Study," Circulation, vol. 103, no. 9, pp. 1245-1249, 2001.

[29] M. Nagai, S. Hoshide, J. Ishikawa, K. Shimada, and K. Kario, "Visit-to-visit blood pressure variations: new independent determinants for carotid artery measures in the elderly at high risk of cardiovascular disease," Journal of the American Society of Hypertension, vol. 5, no. 3, pp. 184-192, 2011.

[30] W. J. Youden, "Index for rating diagnostic tests," Cancer, vol. 3, no. 1, pp. 32-35, 1950.

[31] Y. T. Van der Schouw, A. L. M. Verbeek, and J. H. J. Ruijs, "ROC curves for the initial assessment of new diagnostic tests," Family Practice, vol. 9, no. 4, pp. 506-511, 1992.

[32] R. M. O’Brien, "A caution regarding rules of thumb for variance inflation factors," Quality and Quantity, vol. 41, no. 5, pp. 673690, 2007.

[33] G. Mancia, "Prognostic value of long-term blood pressure variability: the evidence is growing," Hypertension, vol. 57, no. 2, pp. 141-143, 2011.

[34] A. Vasheghani-Farahani, K. Hosseini, H. Ashraf et al., "Correlation of ankle-brachial index and peripheral artery disease with the status of body fat deposition and metabolic syndrome in asymptomatic premenopausal women," Diabetes \& Metabolic Syndrome: Clinical Research \& Reviews, 2016.

[35] J. Solanki, A. Makwana, H. Mehta, P. Gokhale, and C. Shah, "Is the peripheral arterial disease in low risk type 2 diabetic patients influenced by body mass index, lipidemic control, and statins?" Journal of Pharmacology and Pharmacotherapeutics, vol. 7, no. 2, pp. 87-92, 2016.

[36] G. Mancia, F. Messerli, G. Bakris, Q. Zhou, A. Champion, and C. J. Pepine, "Blood pressure control and improved cardiovascular outcomes in the international verapamil SR-trandolapril study," Hypertension, vol. 50, no. 2, pp. 299-305, 2007.

[37] P. Muntner, E. B. Levitan, C. Joyce et al., "Association between antihypertensive medication adherence and visit-to-visit variability of blood pressure," Journal of Clinical Hypertension, vol. 15, no. 2, pp. 112-117, 2013.

[38] T. Okada, T. Wada, Y. Nagaoka, and Y. Kanno, "Association between visit-to-visit clinic blood pressure variability and home blood pressure variability in patients with chronic kidney disease," Renal Failure, vol. 37, no. 3, pp. 446-451, 2015.

[39] C. Giannattasio, M. Failla, M. Hennig et al., "Different relation between 24-h blood pressure and distensibility at different peripheral arteries. Data from the European Lacidipine Study on Atherosclerosis (ELSA)," Journal of Hypertension, vol. 23, no. 3, pp. 557-562, 2005.

[40] F. W. P. J. van den Berkmortel, M. van der Steen, H. Hoogenboom, H. Wollersheim, H. van Langen, and T. Thien, "Progressive arterial wall stiffening in patients with increasing diastolic blood pressure," Journal of Human Hypertension, vol. 15, no. 10, pp. 685-691, 2001.

[41] N. I. Forbang, M. M. McDermott, Y. Liao et al., "Associations of diabetes mellitus and other cardiovascular disease risk factors with decline in the ankle-brachial index," Vascular Medicine, vol. 19, no. 6, pp. 465-472, 2014.

[42] C. E. Bird, M. H. Criqui, A. Fronek, J. O. Denenberg, M. R. Klauber, and R. D. Langer, "Quantitative and qualitative progression of peripheral arterial disease by non-invasive testing," Vascular Medicine, vol. 4, no. 1, pp. 15-21, 1999.

[43] G. Mancia, G. Parati, M. Hennig et al., "Relation between blood pressure variability and carotid artery damage in hypertension: baseline data from the European Lacidipine Study on Atherosclerosis (ELSA)," Journal of Hypertension, vol. 19, no. 11, pp. 1981-1989, 2001.

[44] S. D. Pierdomenico and F. Cuccurullo, "Prognostic value of white-coat and masked hypertension diagnosed by ambulatory monitoring in initially untreated subjects: an updated meta analysis," American Journal of Hypertension, vol. 24, no. 1, pp. 52-58, 2011.

[45] G. S. Stergiou, K. Asayama, L. Thijs et al., "International Database on Home blood pressure in relation to Cardiovascular Outcome (IDHOCO) Investigators. Prognosis of white-coat and masked hypertension: international Database of Home blood pressure in relation to Cardiovascular Outcome," Hypertension, vol. 63, no. 4, pp. 675-682, 2014.

[46] A. Briasoulis, E. Androulakis, M. Palla, N. Papageorgiou, and D. Tousoulis, "White-coat hypertension and cardiovascular events," Journal of Hypertension, vol. 34, no. 4, pp. 593-599, 2016. 


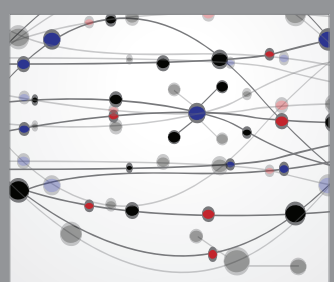

The Scientific World Journal
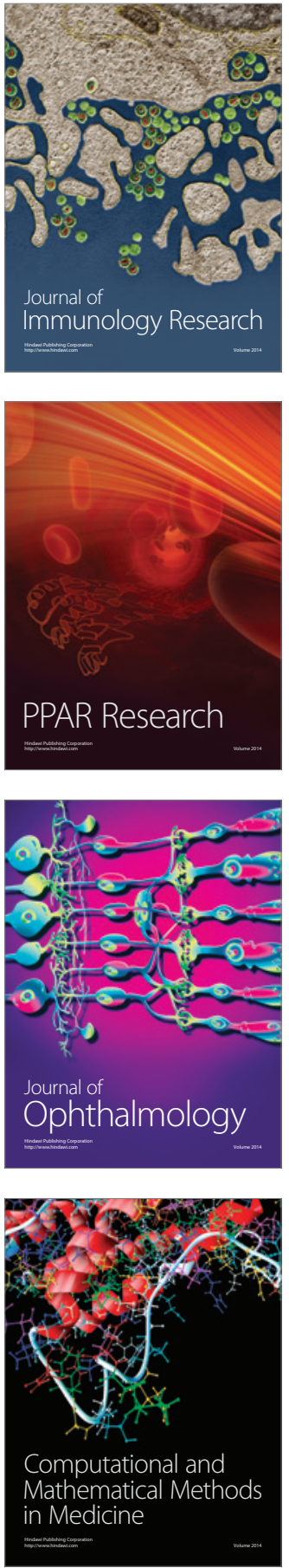

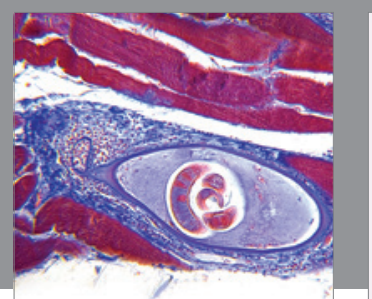

Gastroenterology Research and Practice

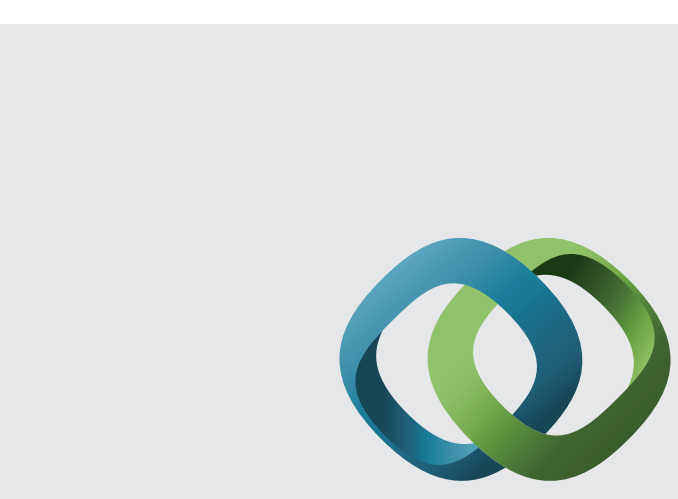

\section{Hindawi}

Submit your manuscripts at

http://www.hindawi.com
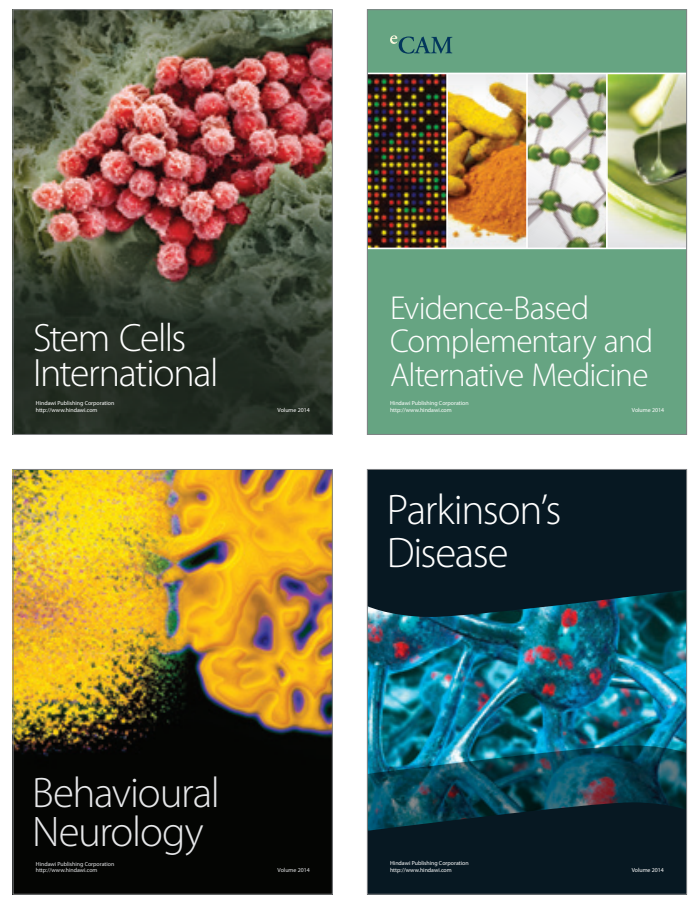
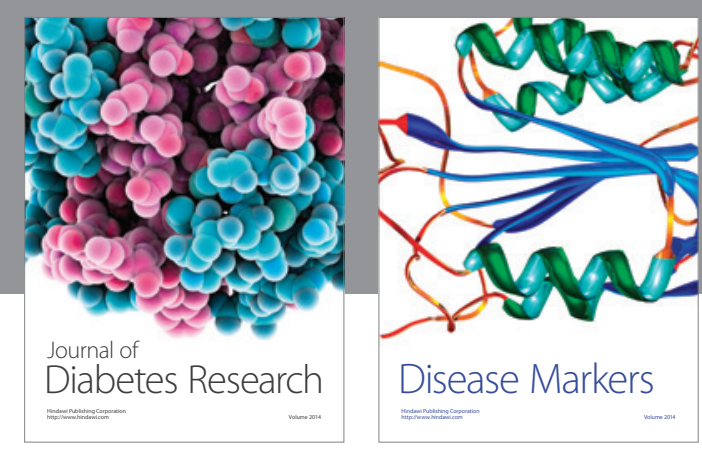

Disease Markers
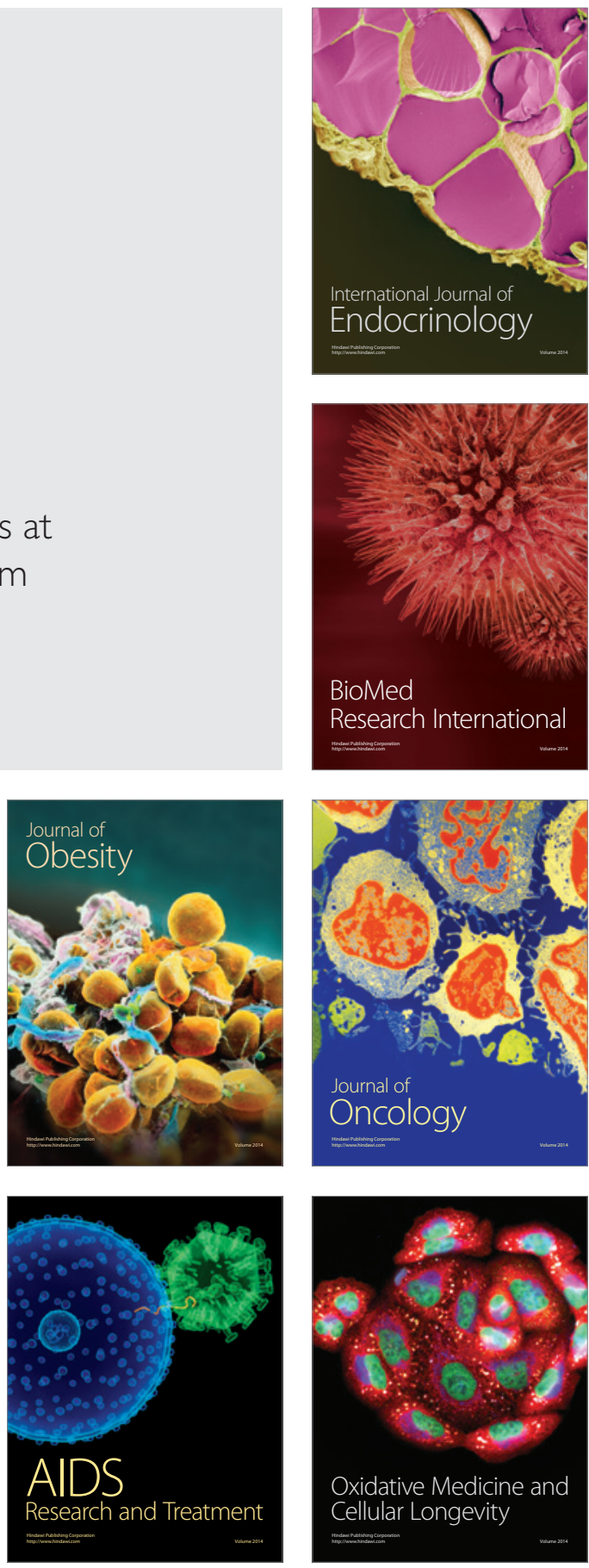